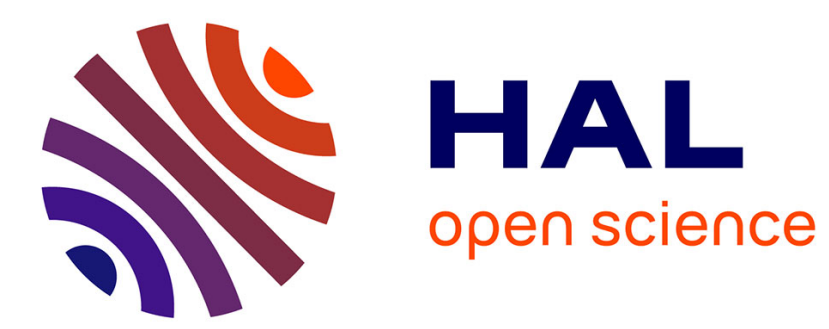

\title{
Timeline Localization
}

Ilona Nawrot, Antoine Doucet

\section{To cite this version:}

Ilona Nawrot, Antoine Doucet. Timeline Localization. 16th International Conference on HumanComputer Interaction (HCII 2014), Jun 2014, Crète, Greece. pp.611 - 622, 10.1007/978-3-319-072333_56. hal-01075063

\section{HAL Id: hal-01075063 https://hal.science/hal-01075063}

Submitted on 16 Oct 2014

HAL is a multi-disciplinary open access archive for the deposit and dissemination of scientific research documents, whether they are published or not. The documents may come from teaching and research institutions in France or abroad, or from public or private research centers.
L'archive ouverte pluridisciplinaire HAL, est destinée au dépôt et à la diffusion de documents scientifiques de niveau recherche, publiés ou non, émanant des établissements d'enseignement et de recherche français ou étrangers, des laboratoires publics ou privés. 


\title{
Timeline Localization
}

\author{
Ilona Nawrot ${ }^{1,2}$ and Antoine Doucet ${ }^{1}$ \\ 1 Normandy University - Unicaen, GREYC, HULTECH, \\ Campus Côte de Nacre, F-14032 Caen, France \\ 2 Poznań University of Economics, WIGE, KEM \\ al. Niepodległości 10, 61-875 Poznań, Poland \\ \{ilona.nawrot, antoine.doucet\}@unicaen.fr
}

\begin{abstract}
The research findings provide evidence that time-oriented data visualizations can contribute to faster information processing, better understanding and improved recall. Thus, they are used in many application domains - medicine, law enforcement, traffic and navigation control to name but a few. Simultaneously, human's time perception varies depending inter alia on culture, language, personal experience and situational factors. Although, the differences caused by the aforementioned aspects were acknowledged and addressed in the Human Computer Interaction (HCI) field for decades their impact on time-oriented data visualizations was largely neglected.

To fill this gap, we investigate the influence of time spatializations (organization of time along axes) on the response time and accuracy of inferences based on time-oriented data visualizations. Moreover, we examine users' preferences toward different time arrangements. Our findings show that user-adapted organization of time along axes can speed up the decision-making process and increase the user experience.
\end{abstract}

Keywords: timeline, localization, time perception, time spatialization, performance, preferences

\section{Introduction}

Despite the importance of time in every aspect of people's lives relatively little attention has been paid to it in the HCI field. Typically it is considered an implicit, yet well defined, variable in the system design. Empirical research investigating cognitive performance of different time-oriented data visualizations, particularly accounting for individual or cultural diversity in time perception, is very limited. Moreover, a number of culture and language related differences in time conceptions and representations were not addressed at all.

In this paper the problem of information flow in time-oriented data visualizations is addressed and empirically investigated.

According to comparative linguistics and cognitive psychology studies, in order to conceptualize time, people rely on space. Nevertheless, factors like longterm cultural, linguistic and personal experience, as well as proximal situational context affect the conceptualization process. Consequently, time organization 
along axes in mental and physical time-oriented data visualizations can differ significantly. In particular, people can represent time along horizontal or vertical axis and in both possible directions (it is from left to right or from right to left and from top to bottom or from bottom to top) [1-3].

Due to globalization interdependencies among the markets are growing. The flow of human capital in between countries is either opened or facilitated. Global corporations are being developed. Multicultural research groups are being formed. Simultaneously, owing to the accelerated pace of life, the need of fast decisionmaking is growing. Thus, as a consequence of information overload, the upsurge of reliance on data integration systems is observed. However, software in domains depending heavily on fast time-oriented data analysis (e.g. medicine [4-6], law enforcement $[7,8]$, military $[9,8]$ or air traffic and navigation control [10]) does not account for the fact that people can perceive, process and interact with temporal information differently. In general, it's design to follow western cultural cues and does not allow for the manipulation of time arrangements along axes.

This phenomenon raises the question of whether differences in time organization observed in between cultures affect the performance in the interaction with the system and user experience. In the empirical study presented in this article we examined the inference time and error rate depending on time arrangements in the scenario of simple information retrieval task, as well as users' preferences toward those arrangements. Moreover, based on the obtained results we formulated the recommendations for software and Web developers and designers.

The rest of the paper is organized as follows. First, we give an overview of the related work in Section 2. In Section 3, we describe the methodology of the experiment conducted to evaluate the possible differences in performance and preferences caused by the interaction with different time arrangements in time visualizations. Section 4 details its results whereas Section 5 provides the discussion and the summary of the paper.

\section{Related Work}

This work explores the role of time organization along axes in reasoning process based on time-oriented data visualizations. Time organization along axes depends on the culture and language experience. Further, it can be also affected by the situational context in which the reasoning takes place [1-3]. Thus, we firstly review the seminal works on localization and cultural adaptivity whose principal goal is to provide users with personalized experience catering their cultural or individual expectations. Then, we discuss the findings of comparative linguistics and cognitive psychology studies on time spatializations. Finally, we survey research on cognitive performance in time-oriented data visualizations.

\subsection{Localization and Cultural Adaptivity}

The minimization of restrictions on access to markets brought about increased competition. The amount of products that is aimed at international audience is 
constantly growing $[16,11]$. This gives more choice to consumers. Hence, businesses to be competitive must meet the needs and expectations of customers to the highest extent. Nevertheless, cross-cultural differences can hinder achieving this goal. Such variations can impact user performance in the interaction with a product, his/her satisfaction and finally its acceptance. In the HCI field, these factors were particularly analyzed in the context of applications or Web sites implementation and design [11-16].

A great deal of research has been focused on the identification of cultural markers, it is elements typical of members of a given culture and likely affecting their preferences. It was demonstrated that software and Web designers should be aware of inter alia colors and fonts usage; wording of warning, error and help messages; or menus' architecture $[12,11,13,16]$. Thus far, the following temporal cultural markers have been distinguished and examined: 1) date and time format $[16] ; 2$ ) style of time management (mono-chronic culture ${ }^{3}$ versus poly-chronic culture ${ }^{4}$ ) [17]; 3 ) time orientation (importance people attach to the past, present and future) [19]; 4) long-term or short-term orientation (degree to which people in a given culture are willing to defer present gratification for the sake of greater future reward) [18].

The knowledge on cultural differences stemmed from the research on cultural markers and it is used to adapt software and Web sites (on both functional and presentation level). Initially, the adaptation was being achieved through largely manual localization process. Firstly, during internationalization phase, culturally sensitive elements were being identified, isolated and all the preparatory tasks facilitating their subsequent adaptation were being performed (e.g. text containers' dimensions had to account for varying translations lengths). Then, during localization phase, the culture-specific content was being infused into previously isolated elements $[20,16]$. Recently, due to expensiveness and high time consumption of manual localization, culturally adaptive systems are being proposed [12]. Such systems using a provided knowledge base and artificial intelligence techniques (usually ML or PGM modeling) automatically create initial user model and adapt culturally sensitive elements accordingly. Then, they analyze users' behavior and refine his/her model. Hereby, they also account for needs of culturally ambiguous individuals [12].

\subsection{Time Spatialization}

Despite extensive research and widespread interest in cross-cultural differences, including aforementioned temporal cultural markers, still a great deal of findings

\footnotetext{
${ }^{3}$ Mono-chronic cultures (e.g. Germany, Japan, most parts of the United States) are characterized by the tendency to handle tasks sequentially. Their members appreciate time and treat time commitments very seriously, strongly adhering to schedule [17].

${ }^{4}$ Multitasking and flexible plans are indicative of the poly-chronic cultures (e.g. China, Mexico, India). Individuals belonging to such cultures view time commitments as goals that can be easily changed depending on the situation [17].
} 
from related domains were not evaluated in the HCI context. This applies particularly to the studies exploring the concept of time and especially the way people spatialize time. Comparative linguistics and cognitive psychology research has revealed an extensive flexibility, as well as cultural and linguistic variability in the way people perceive and conceptualize time. People differ inter alia in whether they see time as limited or open-ended; as static or dynamic with respect to an observer (ego-moving or time-moving metaphor) or moving independently of any observer (time reference point or time-RP metaphor). Moreover, some tend to see time as moving along horizontal axis while others along vertical one $[1,3]$. The axis can also be oriented in various directions (e.g. from bottom-to-top in Zapotec [3] or from east-to-west in Pormpuraaw [1]). In this paper we focus on cross-cultural differences in the space-time mappings and people's preferences with respect to those conceptualizations.

Previous work has established that although wide diversity can be observed between cultures in the way people spatialize time, three main classes of time arrangements can be distinguished $[1]^{5}: 1$ ) along horizontal axis from left to right or 2) from right to left and 3) along vertical axis from top to bottom. First, typical of languages written from left to right (e.g. English), places past on the left side and future on the right side of the horizontal axis $[1,3,23,24,26$, 27]. Second, common among languages written from right to left (e.g. Arabic), locates time on the horizontal axis but past on its right side and future on its left side [24-27]. Third, observed in languages traditionally organizing text in columns (e.g. Mandarin Chinese), position past on the top and future on the bottom of the vertical axis $[2,21-23]$.

Although language and in particular the writing direction are used as an organizing structure for time-space mappings presentation, those are neither the only nor the deciding factors affecting the choice of spatialization. Thus far, the following factors influencing the specific time arrangement along axis activation have been distinguished and acknowledged by the scientific community $[1,22$, 2]: 1) spatiotemporal metaphors (e.g. Christmas is quickly approaching, The meeting has been moved forward two days or He left sad memories behind [3, $2])$; 2) spatial representations available to co-opt with time; 3) organizational patterns in cultural artifacts (e.g. writing direction, clocks, calendars, gestures); 4) cultural or personal experience and dispositions; 5) context.

The aforementioned factors were distinguished based on the studies analyzing free productions, images' arrangements, response time and accuracy in different conditions. Alternative spatializations were proven to be generally available (yet sometimes very rarely used) in each culture. Nevertheless, differences in performance (usually measured by response time in various temporal reasoning tasks) can be observed depending on the salience of the chosen time representation in the given culture ${ }^{6}$.

\footnotetext{
${ }^{5}$ Classification based on the number of people using the given time spatialization frequently.

${ }^{6}$ It was proven that bilinguals can possess two mental timelines. In their case differences in performance are not statistically significant [2].
} 
Finally, the consensus on the mechanism through which the abstract notion of time is conceptualized still has not been reached and is further researched.

\subsection{Cognitive Performance in Time-Oriented Data Visualization}

Time, and particularly the timeline metaphor, is an omnipresent organizational structure used in data visualizations [28]. It is established in scientific literature that time-oriented data visualizations can increase information retrieval performance, aid memorization and insight discovery processes. Nevertheless, studies investigating the aforementioned potential benefits of time visualizations are very sparse and limited.

Thus far, it was demonstrated that visuo-spatial timeline (ViST) format ${ }^{7}$ outperforms both: traditional alphanumeric timeline (AnT) format ${ }^{8}$; and tablebased data visualizations; in terms of reaction and task completion times [29, 30]. It was also shown, that in order to visualize space, time and agents, matrix representation with time as rows or columns, and space and agents as either entries or remaining axis (rows or columns) ${ }^{9}$ is the most intuitive and effective model choice [31]. Moreover, the timeline metaphor was proven advantageous in reducing the first impression biases by increasing the accuracy of initial situation judgments [30]. Further, it was determined that it can mitigate the negative impact of large number of objects to monitor on response time and accuracy [9]. Finally, timelines and particularly timelines annotated with public or personal landmark events were found to benefit both the free and cued recall processes [30, $32,29]$.

Although pointing out numerous advantages of time-oriented data visualizations, the results of the presented research studies are very constrained. Firstly, they do not account for differences in time perception and conceptualizations. Secondly, the research hypotheses are tested on a very specific, homogeneous sample (usually western undergraduate or graduate students) what makes the generalization of the results to the population questionable. Thirdly, comparative studies analyzing the differences in cognitive performance in between various types of time-oriented data visualizations are very limited. Thus, further research in this direction is indispensable.

\section{Methodology}

The aim of this paper is to evaluate whether time spatializations in data visualizations can affect the cognitive performance and satisfaction of individuals exposed to different linguistic or cultural norms. Thereby, whether time organization along axes adaptation should be incorporated as a standard feature in visualizations. An experiment was designed in order to investigate the effects

\footnotetext{
${ }^{7}$ Based on parallel, horizontal time lines.

${ }^{8}$ Based on vertical, subsequent lists of events.

${ }^{9}$ Depending on which dimension was ascribed to time.
} 
of time arrangements along axes on user cognition and experience. It involved temporal reasoning over simple schedules visualizations.

The benefit of time organization along axes adaptation was hypothesized to be two-fold. Firstly, it was expected that the interaction with preferred time arrangement along axes, compared with other well known and frequently used by a user arrangement, would elicit faster reaction time. Secondly, it was assumed that such arrangements in juxtaposition with not-adapted ones would increase the overall user experience. In line with previous comparative linguistics and cognitive psychology findings, the accuracy of inferences was predicted not to be affected by time spatialization manipulations.

Participants. Ninety individuals who reported being fluent in two languages with different most salient time spatializations, yet not being bilingual in those, were recruited to participate in the experiment. Fifty-six of them were male, 34 female, ranging in age from 15 to 55 (median $=28 ; \sigma=7$ ). The subjects represented 18 nationalities, were currently living in 15 countries and were speaking 21 different languages. Thus, their cultural and linguistic backgrounds varied. Over $84 \%$ of the participants already graduated from a university (about $61 \%$ receiving bachelor, $17 \%$ master and $4 \%$ doctoral degree). All but one of them, who reported using the Internet only on weekdays, were using computer and Internet on a daily basis.

Materials. The target stimuli comprised 2 sets of schedules of four individuals depicted on the common matrix-based visualization. Space and agents variables were categorical and both assumed 4 different values: dorm, library, bookstore, gym and Justin, Alex, Sammy, David respectively. Time in the experiment was mapped on the 4-valued ordinal variable taking the morning, noon, afternoon and evening values. Locations and times were represented either as columns or as rows, whereas agents were encoded via 4 -colored $\operatorname{dots}^{10}$. For each set of schedules 28 true-false statements requiring temporal reasoning were being displayed under the matrix (Fig. 1). Within each set, half of the utterances were true. The number of syllables in each query was equated to a high degree. The statements' wording in both sets was similar to a great extent. Both, visualizations and questionnaires, were devised by Kessell [33,31].

Design. The experiment was a within-subjects design with time arranged along different axes in the matrix-based schedule visualization as the main experimental factor. The effectiveness of inferences (response time and number of correct answers) was measured based on the questionnaires developed by Kessell [33,

${ }^{10}$ Lines representation was found to be advantageous in inferences requiring time sequences analysis and in time trends identification tasks. Nevertheless, the participants of the ramp-up phase of the experiment reported to be confused at first once given 2 representations. Since, it could introduce differences in response time due to learning effects, only dots representation was used in the final experiment set up. 


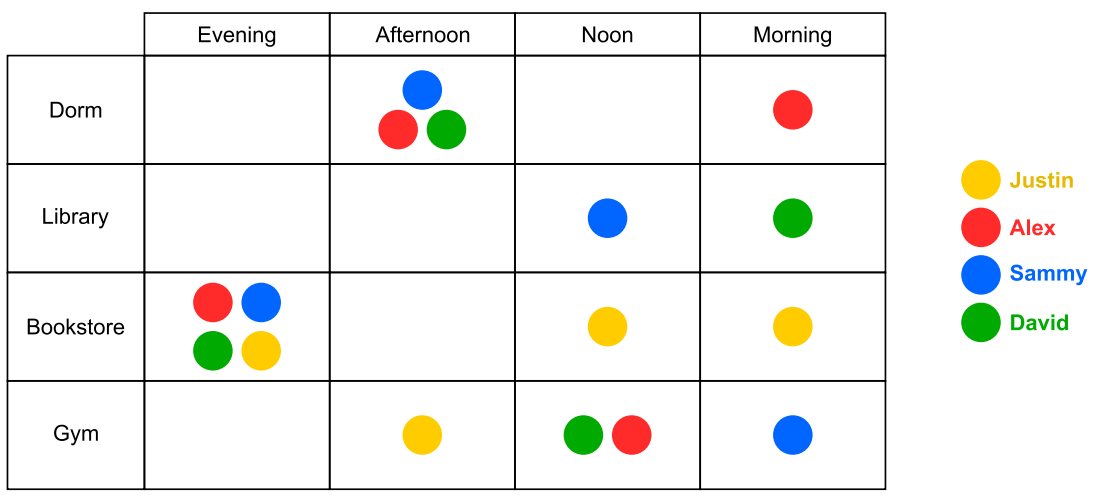

David was in the dorm in the evening.

Fig. 1. Example user stimuli

31]. The users' preferences toward different time arrangements were assessed via self-developed 7-point Likert scale, where 7 represented time spatialization typical of the participant's mother tongue whereas 1 was mapped to the one common in the other language respondent reported was fluent in. Additionally, open question allowed the participants to indicate the conscious reasons behind their time spatialization choice. Finally, the information on participants' performance expectancy was collected using Unified Theory of Acceptance and Use of Technology (UTAUT) [34] which was previously validated cross-culturally [35, 36]. The overview of used assessment metrics is presented in Tab. 1.

Table 1. Summary of evaluation methods used in the experiment

\begin{tabular}{ll}
\hline Attribute & Assessment Method \\
\hline Usability & Overall task completion time \\
& Geometric mean of task completion time [41] \\
& Number of correct answers \\
& 4-item usability scale on a 7-point Likert scale on performance ex- \\
& pectancy [34] \\
& 2-item preferences scale on a 7-point Likert scale ${ }^{\mathrm{b}}$ \\
& open question
\end{tabular}

a indirect measure

${ }^{\mathrm{b}}$ direct measure

The study was run in an online scenario. The participants were recruited via CrowdFlower (a crowdsourcing platform) or Facebook advertisement. The experiment design accounted for the suggestions on conducting human subjects experiments on online labor markets developed by Komarov [39]. Due to insufficient diversity in input device usage (not enough observation to counterbalance), only data collected from subjects using mice as pointing device were considered 
in the analysis. Further, the results collected from participants who reported: 1) having disability or technical problem potentially impairing the performance in the experiment; 2) sleeping less than 6 hours a night before [37, 38]; 3) drinking alcohol or taking drugs (e.g. strong painkillers) 24 hours before the survey; were automatically excluded from the analysis as all of the above listed factors can negatively impact the cognitive performance. Technical requirements of the experiment were reduced to the modern browser installation which is also necessary to run any crowdsourcing platform and thus implicitly assured.

Procedure. Each experiment session comprised three phases: 1) introduction; 2) usability test; and 3) preferences test. In the introduction phase, participants were explained the test routine and in order to proceed, they were required to accept the informed consent form. In addition, demographic, cultural and linguistic background, as well as computer literacy information were collected at this stage.

The second phase started with the group assignment questionnaire establishing participant's language and time spatialization preferences and further checking for factors potentially affecting cognitive performance. Each usability test consisted of 2 blocks of 28 trials. In the congruent scenario (CS) block, subjects interacted with visualization in which time was organized according to his/her reported preferences. In the incongruent (IS) one the other, familiar to him/her time arrangement was used to provide structure to the visualization. In both scenarios, participants were given 1 matrix-based schedule visualization. On each trial, a statement requiring true-false temporal judgment based on the provided visualization was shown under the matrix. Participants were instructed to answer the questions as quickly, yet as accurately, as possible. They were asked to indicate their responses by clicking True or False button.

The usability test was preceded by 4 trials training block assuring the comprehension of the instructions. Scenarios' order was counterbalanced across participants to minimize the learning effects. To avoid ordering effects, the sequence of questions within each scenario was randomized. Furthermore, the participants were tested entirely in English to reduce the effects of proximal language context $[23,2,26]$. The choice of English was motivated by the fact that still a great deal of popular software and Web pages are available only in English. Moreover, it is used as a lingua franca among people working in multicultural environments.

Finally, in the third phase, participants preferences toward different time spatialization survey was carried out.

\section{Analysis and Results}

Data collected from the crowdsourcing platforms are susceptible to the extreme outliers problem [39]. To reduce the impact of abnormal observations on the final results, a method based on the inter-quartile ranges was applied [39]. It detected no extreme outliers, thus no data were excluded from the further analysis. 
Visual inspection of data (histograms, normal Q-Q plots, box plots) indicated that overall completion time and number of errors samples are strongly right skewed which is consistent with other literature findings [40, 41]. The formal inspection of data using Shapiro-Wilk's and Anderson-Darling's tests $(p<0.001$ for all variables) confirmed this hypotheses. Thus, the assumption of normal distribution of data was rejected. Since the assumption of normality was not met, the nonparametric Wilcoxon Signed-Rank Test for matched pairs were used to test the one-sided hypotheses of the superiority of adapted time visualizations over non-adapted ones. Moreover, the mean completion time metric was replaced with the geometric mean completion time, as the latter was proven to be a much more powerful estimate of the population center in skewed samples. All $p$-values (including non-significant ones) were adjusted using Benjamini-Hochberg correction to account for multiple hypotheses testing.

The results indicate that even though, as expected, the accuracy of inferences is not affected by time arrangement changes, the response time is (see Tab. 2).

Table 2. Usability results

\begin{tabular}{lcccc}
\hline Measure & Congruent scenario & Incongruent scenario & $p$-value \\
\hline Overall task completion time & + & - & 0.0002 \\
Geometric mean completion time & + & - & 0.0097 \\
Number of correct answers & $=$ & $=$ & 0.7076 \\
\hline
\end{tabular}

Cronbach's alpha was used to test the Likert scale items' internal consistency and thus overall scales' reliability. Both scales were found to be valid, with excellent $\alpha$ scores about $90 \%$. Therefore, the average overall ratings were computed for them (see Tab. 3). The results reveal strong preferences toward the adapted time arrangement. Interestingly, the preferences could rarely be correlated with the participant's mother tongues. The primary language of education appears to be a much better heuristic.

Table 3. Scales preferences results

\begin{tabular}{lcc}
\hline Likert scale & Cronbach's alpha & Average overall rating \\
\hline Performance expectancy (UTAUT) & 0.886 & $23.71 / 28^{\mathrm{a}}$ \\
Preferences scale & 0.900 & $11.97 / 14^{\mathrm{a}}$ \\
\hline
\end{tabular}

${ }^{\text {a }}$ preferences toward congruent time arrangement

The qualitative analysis of users' comments revealed: 1) decreased cognitive load and relatively greater ease in data decoding, interpretation and understanding (29 indications); 2) routines or cultural customs (13 indications); 3) habits established as part of education process (5 indications); 4) intuitiveness and 
naturalness (4 indications); as the main conscious reasons explaining their preferences.

\section{Conlusions and Future Work}

The evidence provided in this paper extends and partially contradicts the findings of Kessell [31]. It shows that time arrangement along axes can significantly influence performance and user experience in the interaction with time-oriented data visualizations. Consequently, we argue to enrich the time-oriented data visualizations with features allowing time spatialization adaptation and changes. We particularly recommend their inclusion in systems requiring fast information processing (e.g. medical, military systems), as well as in systems underlying on user engagement (e.g. personal storytelling, narrative visualizations). Although, present results demonstrate the impact time spatialization can have on visualizations, they open also many avenues for future research. It includes its influence on information recall and cumulative effects of more complex tasks.

Acknowledgments. This work has been supported by The French Ministry of Foreign Affairs through the Eiffel Excellence Scholarship.

\section{Appendix}

The questionnaires and data collected during the experiment are available to the scientific community and can be accessed at:

https://nawrot.users.greyc.fr/resources/.

\section{References}

1. Boroditsky, L.: How Languages Construct Time. In: Dehaene S., Brannon E. (eds.) Space, time and number in the brain: Searching for the foundations of mathematical thought, pp. 333-341. Academic Press (2011)

2. Miles, L.K., Tan, L., Noble, G.D., Lumsden, J., Macrae, C.N.: Can a mind have two time lines? Exploring space-time mapping in Mandarin and English speakers. Psychon. Bull. Rev. 18(3), 598-604 (2011)

3. Santiago, J., Román, A., Ouellet, M.: Flexible foundations of abstract thought: A review and a theory. In: Maass, A., Schubert, T. (eds.) Spatial dimensions of social thought, pp. 39-108. DE GRUYTER (2011)

4. Rind, A., Wang, T.D., Wolfgang, A., Miksch, S., Wongsuphasawat, K., Plaisant, C., Shneiderman, B.: Interactive Information Visualization to Explore and Query Electronic Health Records. Foundations and Trends in Human-Computer Interaction 5(3), 207-298 (2011)

5. Park, H., Choi, J.: V-Model: A New Innovative Model to Chronologically Visualize Narrative Clinical Texts. In: Konstan, J.A., Chi, E.H., Höök, Ch. (eds.) Proc. of the SIGCHI Conf. on Human Factors in Computing Systems, pp. 453-462. ACM, New York, NY (2012) 
6. Wang, T.D., Deshpande, A., Shneiderman, B.: A Temporal Pattern Search Algorithm for Personal History Event Visualization. IEEE Transactions on Knowledge and Data Engineering 24(5), 799-812 (2012)

7. Kwon, B.Ch., Javed, W, Ghani, S., Elmqvist, N., Yi, J.S., Ebert, D.: Evaluating the Role of Time in Investigative Analysis of Document Collections. IEEE Transactions on Visualization and Computer Graphics 18(11), 1992-2004 (2012)

8. Eppler, M.J., Pfister, R.: Best of Both Worlds: Hybrid Knowledge Visualization in Police Crime Fighting and Military Operations. In: Lindstaedt, S., Granitzer, M. (eds.) Proc. of the 13th Int. Conf. on Knowledge Management and Knowledge Technologies, pp. 17:1-17:8. ACM, New York, NY (2013)

9. Willis, R.A.: Effect of Display Design and Situation Complexity on Operator Performance. In: Proc. of the Human Factors and Ergonomics Society 45th Ann. Meeting, pp. 346-350. Human Factors and Ergonomics Society, Santa Monica (2001)

10. Cordeil, M., Hurter, Ch., Lesbordes, R., Letondal, C., Vinot, J.-L., Conversy, S.: Tangible Encoding of Temporal Data in Air Traffic Control, http://chi2013time.wordpress.com/accepted-papers/ (2013)

11. Su, Y., Liu, D., Yuan, X., Ting, J., Jiang, J., Wang, L., Gao, L.: Webpage Designs for Diverse Cultures: An Exploratory Study of User Preferences in China. In: Kotz P., Marsden G., Lindgaard G., Wesson J., Winckler M. (eds.) Human-Computer Interaction - INTERACT 2013, pp. 339-346. Springer, Heidelberg (2013)

12. Reinecke, K., Bernstein, A.: Improving Performance, Perceived Usability, and Aesthetics with Culturally Adaptive User Interfaces. ToCHI 18(2), A:1-A:29 (2011)

13. Marcus, A., Baumgartner, V.-J.: A Practical Set of Culture Dimensions for Global User-Interface Development. In: Masoodian, M., Jones, S., Rogers, B. (eds.) Computer Human Interaction. LNCS, vol. 3101, pp. 252-261. Springer, Heidelberg (2004)

14. Barber, W., Badre, A.: Culturability: The Merging of Culture and Usability. In: Proc. of the 4th Conf. on Human Factors and the Web (1998)

15. Evers, V., Day D.: The Role of Culture in Interface Acceptance. In: Howard, S., Hammond, J., Lindegaard, G. (eds.) Proc. of the IFIP TC13 Int. Conf. on HumanComputer Interaction. INTERACT, pp. 260-267. Chapman \& Hall, London (1997)

16. Russo, P., Boor, S.: How fluent is your interface? Designing for international users. In: Ashlund S., Mullet K., Henderson A., Hollnagel E., White T. (eds.) Proc. of the INTERACT '93 and CHI '93 Conf. on Human Factors in Computing Systems, pp. 342-347. ACM, New York, NY (1993)

17. Hall, E.T., Hall, M.R.: Understanding Cultural Differences: Germans, French and Americans. Intercultural Press, Boston (1990)

18. Hofstede, G.: Culture's Consequences: Comparing Values, Behaviours, Institutions and Organizations across Nations, 2nd ed. Sage Publications, Thousand Oaks (2001)

19. Adler, N.J., Gundersen, A.: International Dimensions of Organizational Behavior, 5th ed. Thompson South-Western, Mason (2007)

20. De Troyer, O., Casteleyn, S.: Designing Localized Web Sites. In: Zhou, X., Su, S., Papazoglou, M.P., Orlowska, M.E., Jeffery, K. (eds.) WISE 2004. LNCS, vol. 3306, pp. 547-558. Springer, Heidelberg (2004)

21. Boroditsky, L.: Does language shape thought? Mandarin and English speakers' conceptions of time. Cogn. Psychol. 43(1), 1-22 (2001)

22. Boroditsky, L., Fuhrman, O., McCormick, K.: Do English and Mandarin speakers think about time differently? Cognition 118(1), 123-129 (2011)

23. Fuhrman, O., McCormick, K., Chen, E., Jiang, H., Shu, D., Mao, S., Boroditsky, L.: How Linguistic and Cultural Forces Shape Conceptions of Time: English and Mandarin Time in 3D. Cogn. Sci. 35(7), 13051328 (2011) 
24. Fuhrman, O., Boroditsky, L.: Cross-cultural differences in mental representations of time: evidence from an implicit nonlinguistic task. Cogn. Sci. 34(8), 1430-1451 (2010)

25. Nunez, R.E., Sweetser, E.: With the Future Behind Them: Convergent Evidence From Aymara Language and Gesture in the Crosslinguistic Comparison of Spatial Construals of Time. Cogn. Sci. 30(3), 401-450 (2006)

26. Ouellet, M., Santiago, J., Israeli, Z., Gabay, S.: Is the Future the Right Time? Exp. Psychol. 57(4), 308-314 (2010)

27. Tversky, B., Kugelmass, S., Winter, A.: Cross-Cultural and Developmental Trends in Graphic Productions. Cogn. Psychol. 23(4), 515-557 (1991)

28. Aigner, W., Miksch, S., Schumann, H., Tominski, C.: Visualization of TimeOriented Data. Springer-Verlag, London (2011)

29. Bahr, G.S., Walwanis, M.M., Wheeler Atkinson, B.F.: Musically Inspired Computer Interfaces: Reaction Time and Memory Enhancements in Visuo-Spatial Timelines (ViST) for Graphic User Interfaces. In: Stephanidis, C., Antona M. (eds.) UAHCI/HCII, Part II. LNCS, vol. 8010, pp. 555-564. Springer, Heidelberg (2013)

30. Alonso, D.L., Rose A., Plaisant, C., Norman, K.L.: Viewing personal history records: A comparison of Tabular format and graphical presentation using LifeLines. Behaviour \& Information Technology 17(5), 249-262 (1998)

31. Kessell, A., Tversky, B.: Visualizing space, time, and agents: production, performance, and preference. Cognitive Processing 12(1), 43-52 (2011)

32. Ringel, M., Cutrell, E., Dumais, S.T., Horvitz, E.: Milestones in Time: The Value of Landmarks in Retrieving Information from Personal Stores. In: Rauterberg, M., Menozzi, M., Wesson, J. (eds.) Human-Computer Interaction - INTERACT, pp. 184-191. IOS Press (2003)

33. Kessell, A.M.: Cognitive Methods for Information Visualization: Linear and Cyclical Events. PhD Dissertation (2008), https://www.stanford.edu/dept/psychology/ cgi-bin/drupalm/system/files/Kessell_Dissertation.08.pdf

34. Venkatesh, V., Morris, M.G., Davis, G.B., Davis, F.D.: User acceptance of information technology: toward a unified view. MIS Quarterly 27(3), 425-478 (2003)

35. Venkatesh, V., Zhang, X.: Unified Theory of Acceptance and Use of Technology: U.S. vs. China. J. of Global Information Technology Management 13(1), 5-27 (2010)

36. Oshlyansky, L., Cairns, P., Thimbleby, H.: Validating the unified theory of acceptance and use of technology (UTAUT) tool cross-culturally. In: Ramduny-Ellis D., Rachovides D. (eds.) Proc. of the 21st British HCI Group Ann. Conf. on People and Computers: HCI. . . but not as we know it, pp. 83-86. BCS, Swinton, UK (2007)

37. Killgore, W.D.S., Weber, M.: Sleep deprivation and cognitive performance. In: Bianchi, M.T. (ed.) Sleep Deprivation and Disease. Effects on the Body, Brain and Behavior, pp. 209-229. Springer, New York (2014)

38. Alhola, P., Polo-Kantola, P.: Sleep deprivation: Impact on cognitive performance. Neuropsychiatric Dis. Treat. 3(5), 553-567 (2007)

39. Komarov, S., Reinecke, K., Gajos, K.Z.: Crowdsourcing performance evaluations of user interfaces. In: Bødker, S., Brewster, S., Baudisch, P., Beaudouin-Lafon, M., Mackay, W.E. (eds.) Proc. of the SIGCHI Conf. on Human Factors in Computing Systems, pp. 207-216. ACM, New York, NY (2013)

40. Ratcliff, R., McKoon, G.: The diffusion decision model: theory and data for twochoice decision tasks. Neural Computation 20(4), 873-922 (2008)

41. Sauro, J., Lewis, J.R.: Average task times in usability tests: what to report? In: Proc. of the SIGCHI Conf. on Human Factors in Computing Systems, 2347-2350 (2010) ACM, Atlanta, GA 